Informes de la Construcción

Vol. 66, EXTRA 1, mo14

diciembre 2014

ISSN-L: 0020-0883

doi: http://dx.doi.org/10.3989/ic.13.099

\title{
Armado Óptimo de Secciones de Hormigón Posteso
}

\section{Optimal Reinforcement for Post Stressed Concrete Cross Sections}

\author{
F. A. García-Villena ${ }^{(*)}, \underline{\text { J. F} \text {. Carbonell-Márquez }}{ }^{(*)}$
}

\section{RESUMEN}

Para un rango de valores de área de armadura activa y de excentricidad que cumplen los requisitos de tensión impuestos por el estado límite de servicio, se evalúa la armadura pasiva necesaria en estado límite último mediante la técnica RSD (Reinforcement Sizing Diagrams) teniendo en cuenta la presencia de la armadura activa. Se selecciona la solución que conduce a un valor mínimo del coste total de armado pasivo y activo.

Palabras clave: Hormigón Posteso; vigas; armado óptimo; Reinforcement Sizing Diagrams.

\section{ABSTRACT}

The amount of passive reinforcement to be provided in the ultimate limit state is computed by means of RSD (Reinforcement Sizing Diagrams) taking into account the presence of active reinforcement, for a particular range of pairs active steel area - active steel eccentricity $\left(A_{p}-d_{p}\right)$ that meet the requirements imposed by the serviceability limit state. The solution that provides the minimum total cost of both active and passive steel is selected.

Keywords: Post Stressed Concrete; beams; optimal reinforcement; Reinforcement Sizing Diagrams.

(*) Universidad de Granada, (España).

(**) Universidad Loyola Andalucía, Sevilla (España).

Persona de contacto/Corresponding author: jfcarbonell@uloyola.es (J. F. Carbonell-Márquez)

Cómo citar este artículo/Citation: García-Villena, F. A., Carbonell-Márquez, J. F. (2014). Armado Óptimo de Secciones de Hormigón Posteso. Informes de la Construcción, 66(EXTRA-1): m014, doi: http://dx.doi.org/10.3989/ic.13.099.

Licencia / License: Salvo indicación contraria, todos los contenidos de la edición electrónica de Informes de la Construcción se distribuyen bajo una licencia de uso y distribución Creative Commons Reconocimiento no Comercial 3.o. España (cc-by-nc). 


\section{INTRODUCCIÓN}

El procedimiento de armado de secciones de elementos continuos postesos -con armadura activa adherente- comienza con un predimensionamiento de la fuerza de tesado inicial $P_{o}$ y de su excentricidad $e$ frente al centro de gravedad de la sección más solicitada. Para su determinación, es común el empleo de los denominados diagramas de Magnel. Estos diagramas representan gráficamente las limitaciones de tensiones normales a la sección impuestas en las fibras superior e inferior para el estado de transferencia y el estado de servicio, de tal manera que introduciendo en el gráfico el par $P_{o}-e$ adoptado en el diseño, el ingeniero puede saber si se cumplen dichas prescripciones. Establecido el valor de la fuerza $P_{o}$, el área de armadura activa $A_{p}$ a disponer en la sección se obtiene como:

$$
A_{P}=\frac{P_{\mathrm{o}}}{\sigma_{P_{0}}}
$$

siendo $\sigma_{p 0}$ la tensión inicial de tesado.

Una vez realizado el predimensionamiento de la armadura activa, habrá que realizar las comprobaciones pertinentes en estado límite último, centrándose este trabajo en la resistencia de la sección frente a esfuerzos normales, esto es, se considera la sección sometida a una combinación de fuerza axil y momento flector respecto a su eje fuerte. En numerosas ocasiones, al realizar esta comprobación, resulta necesaria la disposición de armadura pasiva en la sección. Esta armadura pasiva necesaria puede ser calculada empleando cualquiera de los métodos clásicos comúnmente utilizados para el diseño de secciones de hormigón armado sometidas a la combinación de carga axil y momento flector respecto a su eje principal de inercia. Sin embargo, ha surgido en la última década un nuevo enfoque de dicho problema de diseño, presentado por Hernández-Montes et al. (2), denominado RSD -del inglés Reinforcement Sizing Diagrams- y que presenta todas las soluciones posibles para las armaduras superior e inferior. La observación del tipo de soluciones óptimas -mínima cantidad de acero- obtenidas ha conducido al desarrollo del denominado Teorema del Armado Óptimo de Secciones (3) -TOSR, del inglés Theorem of Optimal Section Reinforcement-, el cual ofrece una serie de condiciones a imponer en el diseño para la obtención del armado óptimo de la sección.

Para un elemento de hormigón armado, las combinaciones posibles de armado superior e inferior requeridos para conferir la adecuada resistencia en rotura a la sección sometida a momento flector y a carga axil constituye un conjunto infinito de soluciones entre las que se incluye la solución de armado simétrico obtenida mediante el empleo de los diagramas de interacción $N-M$ clásicos. Mediante la técnica RSD, la fami- lia de soluciones se representa gráficamente en función de la posición de la fibra neutra, $x$, tal y como es explicado por Hernández-Montes et al. (2). En este sentido López-Martín et al. (4) ha desarrollo recientemente un procedimiento basado en la técnica RSD que, dependiendo de la excentricidad equivalente de la carga axil, proporciona cuál de las cinco condiciones dictadas por el TOSR debe ser impuesta para obtener el armado óptimo de la sección.

En el presente trabajo se pretende aplicar el concepto de armado óptimo desde el inicio del proceso de diseño de una sección de hormigón postesado con armadura activa adherente. Considerando la deformación inicial de la armadura activa, el cálculo de la resistencia de la sección en estado límite último de flexión puede ser planteado como el de una sección de hormigón armado convencional. De esta manera la técnica RSD podría ser empleada como hasta ahora proponía Hernández-Montes et al. (2), es decir, las distintas soluciones de armado pasivo superior e inferior seguirían siendo función de la posición de la fibra neutra, $x$. En el caso de las secciones de hormigón postesado, para cada par de valores de $A_{p}-e$ obtenido a partir del diagrama de Magnel, la técnica RSD permitirá determinar el armado pasivo óptimo. Una vez realizado el predimensionamiento de la sección al completo, se pueden evaluar los valores exactos de las pérdidas de la fuerza de postesado y comprobar que las limitaciones relativas a la tensión se cumplen efectivamente.

\section{PREDIMENSIONAMIENTO DE LA ARMADURA ACTIVA DE UNA SECCIÓN: DIAGRAMAS DE MAGNEL}

El hormigón es una material versátil para su uso en elementos estructurales, con una muy buena resistencia a compresión. Por contra, se trata de un material con una pobre resistencia a tracción, por lo que es necesaria la inclusión de elementos de acero para que soporten las tracciones. El uso de cables de acero pretensados o postesados introduce un estado de compresión previa en el elemento estructural de hormigón, de manera que se anulan o disminuyen las tracciones en las distintas etapas de la vida útil de la estructura.

Una vez conocidas las dimensiones de la sección transversal del elemento postesado, es imprescindible determinar el área de armadura activa, su tensión inicial, $\sigma_{p 0}$, que dará como resultado la fuerza de tesado inicial $P_{0}$, y la trayectoria, $e$, de los cordones de armadura activa a lo largo del elemento -Figura 1-. Dado que resulta necesario calcular las pérdidas instantáneas y diferidas de la fuerza de tesado y dichas pérdidas dependen de las características del armado -activo y pasivode la sección, será preciso realizar primero un predimensionamiento de dicho armado y una posterior comprobación de los cálculos realizados.

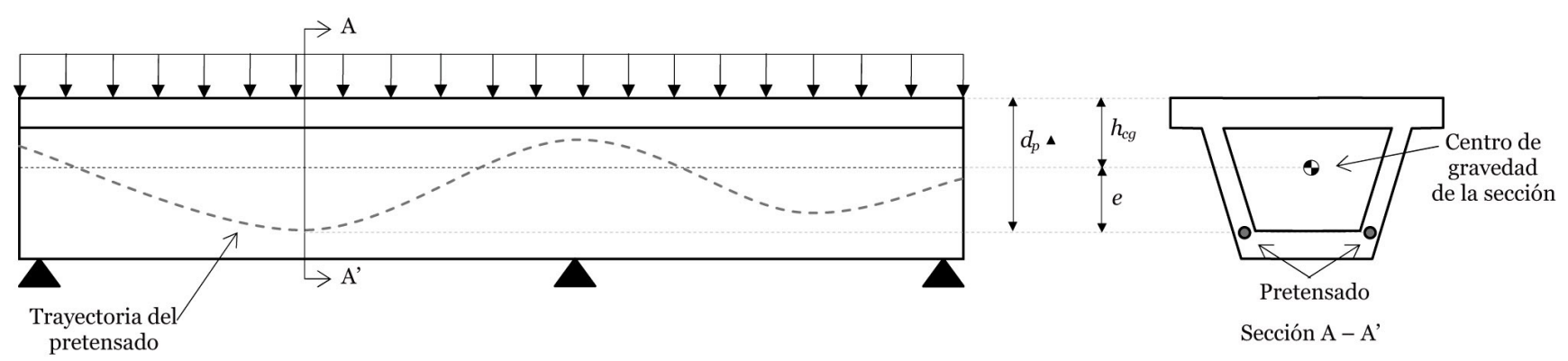

Figura 1. Esquema del problema de diseño de una viga continua de hormigón posteso. 
Debido a las distintas combinaciones de cargas a las que el elemento se ve sometido y a las pérdidas en la fuerza de tesado, la sección ha de soportar diferentes estados de tensión a lo largo de su vida útil. Así pues, a la hora de determinar la armadura activa y su posición en la sección transversal, hay que comprobar que dichos estados de tensión no superan ciertos límites. Dado que en este punto se está realizando un predimensionamiento del armado activo, se considerarán las características brutas de la sección a efectos de cálculo. Además, se considera válida la hipótesis de la proporcionalidad a lo largo del tiempo entre momentos isostáticos e hiperestáticos provocados por efecto de la armadura activa (5).

En el estado de transferencia, es decir, cuando se introduce la tensión en la armadura activa una vez hormigonado el elemento estructural, ha de asegurarse que toda la sección se encuentra comprimida bajo el efecto de la armadura activa y el peso propio del elemento. Así pues, considerando como positivos las deformaciones y tensiones de compresión y los momentos que comprimen la parte superior de la sección, la tensión en la fibra superior debe cumplir:

$$
\sigma_{\text {sup }}=\gamma_{\text {Pdes }} \alpha P_{\mathrm{o}}\left(\frac{1}{A}-\frac{(1-\lambda) e}{I} y_{\text {sup }}\right)+\frac{M_{p p}}{I} y_{\text {sup }} \geq 0
$$

Por su parte, la tensión de compresión en la fibra inferior no debe superar un nivel determinado por el artículo 49.2.1 de la EHE-o8 (6) para evitar fisuras por compresión en el hormigón:

$$
\sigma_{\text {inf }}=\gamma_{P d e s} \alpha P_{\mathrm{o}}\left(\frac{1}{A}+\frac{(1-\lambda) e}{I} y_{\mathrm{inf}}\right)-\frac{M_{p p}}{I} y_{\mathrm{inf}} \leq 0.6 f_{c k, j}
$$

En [2] y [3] $\gamma_{\text {Pdes }}$ es el coeficiente parcial de seguridad en estado límite de servicio para la acción del postesado, de valor 1,10 de acuerdo con la Tabla 12.2 de la EHE-o8 (6); a es el coeficiente que tienen en cuenta la estimación de las pérdidas iniciales, $A$ es el área bruta de la sección, $I$ su momento de inercia, $\lambda$ es la relación entre los momentos hiperestático e isostático ocasionados por la armadura activa -normalmente de distinto sentido-, $y_{\text {sup }}$ e $y_{\text {inf }}$ son las distancias entre las fibras superior e inferior y el centro de gravedad de la sección respectivamente, $M_{p p}$ es el momento provocado por el peso propio y $f_{c k, j}$ es la resistencia característica a compresión del hormigón a fecha de tesado.

Una vez la estructura se encuentra en servicio, hay que comprobar, en función de la clase de exposición de la estructura que se cumplen las prescripciones de tensión y de abertura de fisura dictadas por la normativa de aplicación. La Tabla 1 muestra las prescripciones dadas en la Tabla 7.1N del Euro- código 2 (EC2) (7); esta tabla es equivalente a la Tabla 5.1.1.2 de la EHE-o8 (6).

Así pues, considerando que la estructura a diseñar se encuentra en un tipo de ambiente $\mathrm{XC}_{2}$-equivalente al IIa en la normativa EHE-08 (6)-, es decir, una estructura situada en un ambiente de humedad alta en la que la corrosión de la armadura tiene un origen diferente de los cloruros -ambiente habitual para un puente de carretera-, las prescripciones a cumplir son que bajo la combinación de cargas frecuentes la abertura de fisura ha de ser menor que $0,2 \mathrm{~mm}$ y que se debe producir descompresión bajo la combinación de cargas cuasipermanente. Según el EC2 (7), la condición de descompresión se cumple cuando todas las partes de las armaduras activas o vainas adherentes están situadas al menos $25 \mathrm{~mm}$ dentro del hormigón sometido a compresión.

Aunque estas comprobaciones se realizan bajo las combinaciones de carga cuasipermanente y frecuente y no se realiza ningún cálculo en servicio para la combinación característica, hay que considerar que esta combinación actúa alguna vez a lo largo de la vida útil de la estructura, provocando al fisuración de la sección (5). Por este motivo, las comprobaciones bajo combinación cuasipermanente o frecuente debieran hacerse considerando la sección como fisurada. No obstante, dado que se está realizando un predimensionamiento de la sección, imponer estas condiciones complicaría en exceso el proceso, motivo por el que es recomendable hacer el predimensionamiento con el criterio de que la máxima tracción -en la fibra inferior- no supere el valor de la resistencia característica del hormigón a tracción (8) bajo carga cuasipermanente y comprobar la descompresión de la armadura activa bajo dicha combinación. Además, la condición de que la abertura de fisura resulte menor que $0,2 \mathrm{~mm}$ puede sustituirse, de forma simplificada, por la condición expresada en el comentario al artículo 49.2.4 de la EHE-o8 (6) la cual dicta que si el incremento de tensión de la armadura activa debido a la acción de las cargas exteriores es inferior a $200 \mathrm{~N} / \mathrm{mm}^{2}$, en una sección sin armadura pasiva, puede suponerse que la abertura de fisura es inferior a 0,2 mm. De esta forma, las condiciones a imponer son:

$$
\begin{gathered}
\sigma_{\text {inf }}=\gamma_{P f a v} \beta P_{\mathrm{o}}\left(\frac{1}{A}+\frac{(1-\lambda) e}{I} y_{\text {inf }}\right)-\frac{M_{\text {cuasip }}}{I} y_{\text {inf }} \geq-f_{\text {ctk }}=-0.7 f_{c t m} \\
\sigma_{\varrho(e+25)}=\gamma_{P f a v} \beta P_{0}\left(\frac{1}{A}+\frac{(1-\lambda) e}{I}(e+25 \mathrm{~mm})\right)-\frac{M_{\text {cuasip }}}{I}(e+25 \mathrm{~mm}) \geq 0 \\
\sigma_{p}=\frac{M_{\text {frec }}-M_{p p}}{I} e \frac{E_{p}}{E_{c}}<200 \mathrm{~N} / \mathrm{mm}^{2}
\end{gathered}
$$

Tabla 1. Valores máximos de ancho de fisura, en mm, recomendados por el EC2, Tabla 7.1N.

\begin{tabular}{|c|c|c|}
\hline \multirow{2}{*}{ Clase de exposición } & $\begin{array}{c}\text { Elementos con armadura pasiva y con } \\
\text { armaduras activas no adherentes }\end{array}$ & $\begin{array}{c}\text { Elementos con armaduras activas } \\
\text { adherentes }\end{array}$ \\
\cline { 2 - 3 } & Combinación de cargas cuasipermanente & Combinación de cargas frecuente \\
\hline $\mathrm{Xo}, \mathrm{XC} 1$ & $0,4^{1}$ & 0,2 \\
\hline $\mathrm{XC} 2, \mathrm{XC} 3, \mathrm{XC} 4$ & 0,3 & $0,2^{2}$ \\
\hline $\mathrm{XD} 1, \mathrm{XD} 2, \mathrm{XS} 1, \mathrm{XS} 2, \mathrm{XS} 3$ & & Descompresión \\
\hline
\end{tabular}

NOTA 1 Para clases de exposición $\mathrm{Xo}, \mathrm{XC1}$, la abertura de la fisura no influye en la durabilidad y este límite se establece para proporcionar, en general, una apariencia aceptable. En ausencia de condiciones de apariencia se puede tratar este límite de modo menos estricto.

NOTA 2 Para estas clases de exposición, la descompresión se debería comprobar bajo la combinación cuasipermanente de cargas 
En [4], [5] y [6], $\gamma_{\text {Pfau }}$ es el coeficiente parcial de seguridad en estado límite de servicio para la acción del postesado, de valor 0,90 de acuerdo con la Tabla 12.2 de la EHE-08 (6), $\beta$ es el coeficiente que tiene en cuenta la estimación de las pérdidas a largo plazo en el tesado, $M_{\text {cuasip }}$ y $M_{\text {frec }}$ son los momentos en la sección relativos a las combinaciones cuasipermanente y frecuente, respectivamente y $E_{p}$ y $E_{c}$ son los módulos de elasticidad de la armadura activa y del hormigón, respectivamente.

Dado que estas condiciones presentes en [4], [5] y [6] son simplificaciones de las anteriormente explicadas para facilitar el proceso de predimensionamiento, una vez finalizado éste, deberá comprobarse que las condiciones estrictas se cumplen efectivamente. Además, las condiciones expuestas en las [2] y [3] deberán comprobarse teniendo en cuenta las propiedades geométricas de la sección neta en la aplicación del tesado y el peso propio.

Ahora bien, dado que no se conocen ni la fuerza de tesado ni la posición de la armadura activa, tomando [2] - [5] se pueden obtener una serie de inecuaciones de la forma $1 / P_{0} \leq f(e)$ o $1 / P_{0} \geq f(e)$ y con la Eq. [6] se puede obtener un valor máximo para $e$. Ya que se conoce la tensión inicial de tesado, $\sigma_{p 0}$, el cociente $1 / P_{0}$ puede sustituirse equivalentemente por $1 / A_{p}^{p 0}$ :

$$
\begin{gathered}
\frac{1}{A_{p}} \geq \frac{\sigma_{p \mathrm{o}} \gamma_{\text {Pdes }} \alpha\left(\frac{1}{A}-\frac{(1-\lambda) e}{I} y_{\text {sup }}\right)}{-\frac{M_{p p}}{I} y_{\text {sup }}} \\
\frac{1}{A_{p}} \geq \frac{\sigma_{p \mathrm{o}} \gamma_{\text {Pdes }} \alpha\left(\frac{1}{A}+\frac{(1-\lambda) e}{I} y_{\text {inf }}\right)}{0.6 f_{\text {ck,j }}+\frac{M_{p p}}{I} y_{\text {inf }}} \\
\frac{1}{A_{p}} \leq \frac{\sigma_{p \mathrm{o}} \gamma_{\text {Pfav }} \alpha\left(\frac{1}{A}+\frac{(1-\lambda) e}{I} y_{\text {inf }}\right)}{-0.7 f_{c t m}+\frac{M_{\text {cuasip }}}{I} y_{\text {inf }}} \\
\frac{1}{A_{p}} \leq \frac{\sigma_{p \mathrm{o}} \gamma_{\text {Pfav }} \alpha\left(\frac{1}{A}+\frac{(1-\lambda) e}{I}(e+25 \mathrm{~mm})\right)}{\frac{M_{\text {cuasip }}(e+25 \mathrm{~mm})}{I}(e)}
\end{gathered}
$$

$$
e<\frac{200 \mathrm{~N} / \mathrm{mm}^{2}}{\frac{M_{\text {frec }}-M_{p p}}{I} \frac{E_{p}}{E_{c}}}
$$

\section{CONSIDERACIONES EN EL DISEÑO RESISTENTE DE SECCIONES DE HA EN FLEXIÓN}

El diseño en rotura de una sección de HA sometida a flexión compuesta respecto a su eje principal de inercia conlleva la consideración simultánea de las condiciones de compatibilidad, los modelos constitutivos y las ecuaciones de equilibrio para los materiales -hormigón y aceros pasivo y activo- a nivel de la sección.

\subsection{Hipótesis de Bernouilli y estado límite último de la sección según EC2}

Las condiciones de compatibilidad hacen uso de las hipótesis de Bernouilli que dicta que las secciones planas permanecen planas después de la deformación. Se admite que no existe deslizamiento entre el hormigón y las barras de acero en la sección. La hipótesis de Bernouilli permite que la distribución de deformaciones a lo largo de la sección pueda ser definida mediante dos variables: la deformación en el centro de gravedad de la sección $-\varepsilon_{c g}-\mathrm{y}$ la curvatura $-\phi-$. De este modo, la deformación a cierto nivel $y$ por encima del centro de gravedad de la sección puede ser definida de acuerdo a [12] -Figura 2-:

$$
\varepsilon\left(y, \varepsilon_{c g}, \phi\right)=\varepsilon_{c g}+\phi y
$$

La expresión empleada en [12] considera como positivas la deformación por compresión y la curvatura que produce tracción en la fibra inferior de la sección.

Siguiendo con la hipótesis de Bernouilli, la normativa de aplicación -EHE-o8 (6) y EC2 (7)- propone una serie de rangos de distribuciones de deformaciones de rotura, tal y como la Figura 3 muestra: los planos que pivotan en el punto A se corresponden con distribuciones de deformación para las cuales el acero falla a tracción mientras que los planos que pivotan en los puntos $\mathrm{B}$ o $\mathrm{C}$ se corresponden con el agotamiento del hormigón a compresión. Adoptando el diagrama rectangular de compresiones en rotura para el hormigón, el EC2 (7) considera una deformación de compresión última para flexo compresión, $\varepsilon_{c u 3}$, y para compresión pura, $\varepsilon_{c 3}$. En

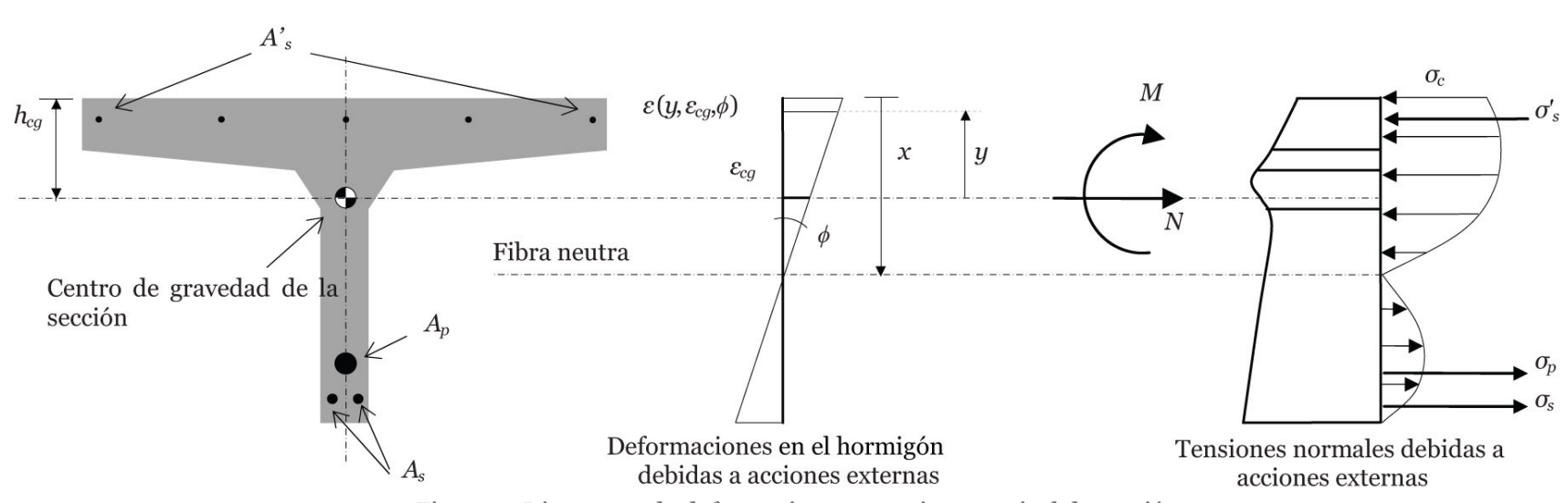

Figura 2. Diagramas de deformaciones y tensiones a nivel de sección. 


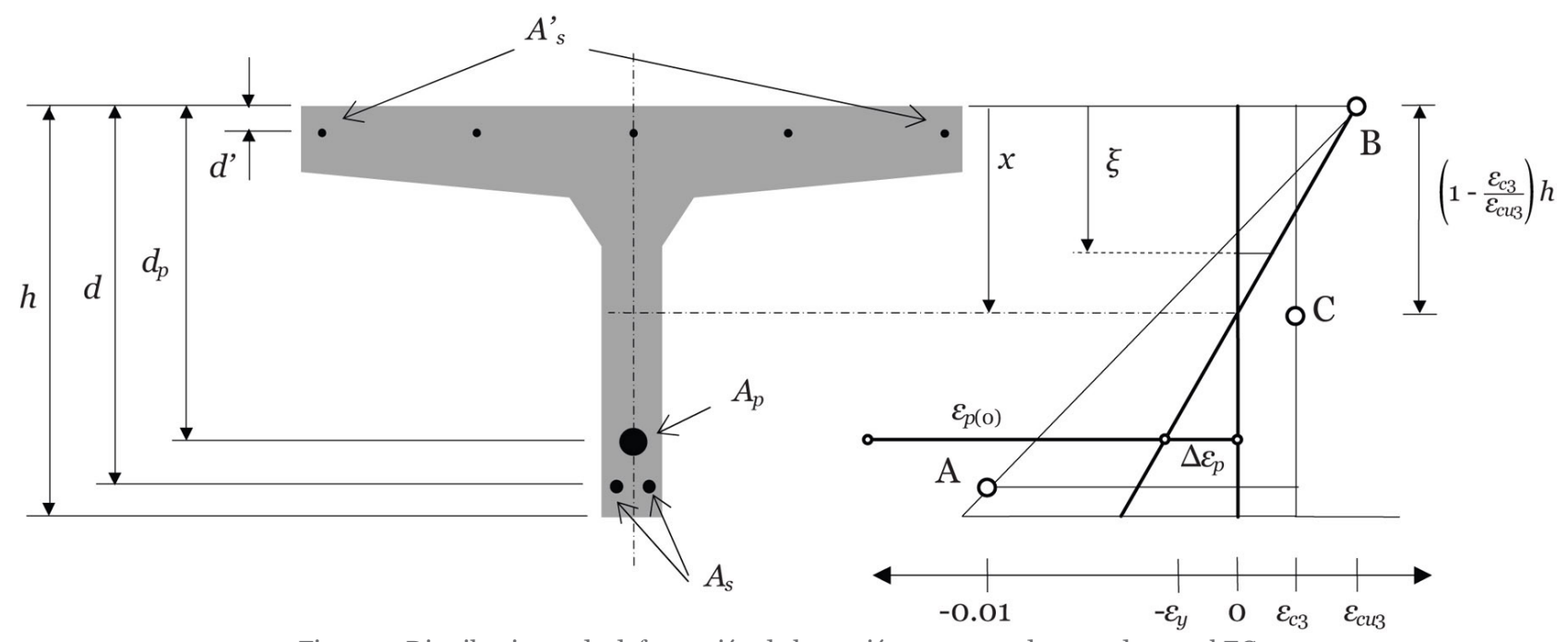

Figura 3. Distribuciones de deformación de la sección en rotura de acuerdo con el EC2.

el presente trabajo se considera que el acero responde a un modelo tensión-deformación bilineal sin endurecimiento, de manera que el EC2 (7) permite que no se imponga ninguna limitación de deformación a tracción y el pivote A en la Figura 3 desaparece. Otra consecuencia es que la posición de la fibra neutra, $x$, toma valores en el intervalo $[0, \infty)$.

De acuerdo con las distribuciones de deformaciones presentadas en la Figura 3, en nuestro caso la deformación de rotura en cualquier fibra situada a una distancia $\xi$ desde la fibra superior de la sección puede ser expresada en términos de la posición de la fibra neutra, $x$, como:

$$
\varepsilon(\xi, x)=\left\{\begin{array}{lll}
\varepsilon_{c u} \frac{x-\xi}{x} & \text { si } & 0 \leq x<h \\
\varepsilon_{c 3} \frac{x-\xi}{x-\Xi} & \text { si } & x \geq h
\end{array}\right.
$$

donde $\Xi=h\left(1-\frac{\varepsilon_{c 3}}{\varepsilon_{c u 3}}\right)$.

\subsection{Modelo constitutivo del hormigón en rotura}

Para el modelo de comportamiento tensión deformación del hormigón se han propuesto numerosas formulaciones a lo largo de la historia (9). Dado que en este trabajo emplea el modelo constitutivo del hormigón a la hora de realizar los diagramas RSD, dicho modelo constitutivo ha de corresponderse con el estado de rotura del hormigón. Tal y como se ha mencionado anteriormente, se adopta el diagrama rectangular de compresiones en rotura para el hormigón propuesto en el EC2 (7). Así pues, los valores de la deformación última son:

- Compresión pura:

$$
\varepsilon_{c 3}(\% \text { o }) \begin{cases}1.75 & \text { para } f_{c k} \leq 50 \mathrm{MPa} \\ 1.75+0.55\left(f_{c k}-50\right) / 40 & \text { para } 50<f_{c k} \leq 90 \mathrm{MPa}\end{cases}
$$

- Flexo compresión:

$$
\varepsilon_{c u 3}(\%) \begin{cases}3.5 & \text { para } f_{c k} \leq 50 \mathrm{MPa} \\ 2.6+35\left(90-f_{c k}\right)^{4} / 100 & \text { para } 50<f_{c k} \leq 90 \mathrm{MPa}\end{cases}
$$

La resistencia de diseño del hormigón a compresión de acuerdo con el EC2 (7) viene dada por:

$$
f_{c d}=\alpha_{c c} \frac{f_{c k}}{\gamma_{c}}
$$

donde $f_{c k}$ es la resistencia característica a compresión de la probeta cilíndrica ensayada a los 28 días, $\alpha_{c c}$ es el coeficiente que considera la reducción de la resistencia a compresión del hormigón a largo plazo y otros efectos desfavorables debidos al modo en que la carga es aplicada cuyo valor debe ser escogido en el rango o,8-1,0 -en este trabajo se adopta $0,85^{-}-\mathrm{y}, \gamma_{c}$ es el factor parcial de seguridad tomado en este trabajo como 1,5 -situaciones de diseño permanentes y transitorias-.

La resultante del bloque de compresiones en el hormigón, $N_{c}$, puede ser definida como una función de la posición de la fibra neutra, $x$, como:

$$
N_{c}(x)=\left\{\begin{array}{lll}
0 & \text { si } \quad x \leq 0 \\
n f_{c d} \int_{0}^{\lambda x} b(\xi) d \xi & \text { si } & 0 \leq x<h / \lambda \\
n f_{c d} \int_{0}^{h} b(\xi) d \xi & \text { si } & x \geq h / \lambda
\end{array}\right.
$$

siendo $b(\xi)$ el ancho de la sección en la fibra situada a $\xi$ de la fibra superior (Figura 4).

Los valores de $\lambda$ y $\eta$ vienen dados por el EC2 (7) de acuerdo a [16]:

$$
\begin{aligned}
& \lambda= \begin{cases}0.8 & \text { para } f_{c k} \leq 50 \mathrm{MPa} \\
0.8-\left(f_{c k}-50\right) / 400 & \text { para } 50<f_{c k} \leq 90 \mathrm{MPa}\end{cases} \\
& \eta= \begin{cases}1.0 & \text { para } f_{c k} \leq 50 \mathrm{MPa} \\
1.0-\left(f_{c k}-50\right) / 200 & \text { para } 50<f_{c k} \leq 90 \mathrm{MPa}\end{cases}
\end{aligned}
$$

\subsection{Modelo constitutivo del acero}

Los modelos tensión-deformación inicialmente considerados tanto para el acero pasivo como para el activo son bilineales 
sin tener en cuenta el endurecimiento por deformación y simétricos. Ya que la presencia del acero dentro del hormigón no fue tenida en cuenta a la hora de formular la resultante de las compresiones en el hormigón -[15]-, los modelos de acero anteriormente mencionados se modifican ligeramente para considerar el citado fenómeno. Así pues, los modelos de tensión -deformación de la armadura pasiva quedan:

$$
\sigma_{s}\left(\varepsilon_{s}\right) \circ \sigma_{s}^{\prime}\left(\varepsilon_{s}\right)= \begin{cases}f_{y d}-\eta f_{c d} & \text { si } \quad \varepsilon_{s} \geq \frac{f_{y d}-\eta f_{c d}}{E_{s}} \\ E_{s} \varepsilon_{s} & \text { si } \quad-\frac{f_{y d}}{E_{s}}<\varepsilon_{s}<\frac{f_{y d}-\eta f_{c d}}{E_{s}} \\ -f_{y d} & \text { si } \varepsilon_{s} \leq-\frac{f_{y d}}{E_{s}}\end{cases}
$$

donde $f_{y d}$ se corresponde con $f_{y} / \gamma_{s}$ y $\gamma_{s}=1,15$. El modelo del acero activo queda:

$$
\sigma_{p}\left(\varepsilon_{p}\right)= \begin{cases}f_{y p d}-\eta f_{c d} & \text { si } \geq \frac{f_{y p d}-\eta f_{c d}}{E_{p}} \\ E_{p} \varepsilon_{p} & \text { si }-\frac{f_{y p d}}{E_{p}}<\varepsilon_{p}<\frac{f_{y p d}-\eta f_{c d}}{E_{p}} \\ -f_{y p d} & \text { si } \varepsilon_{p} \leq-\frac{f_{y p d}}{E_{p}}\end{cases}
$$

donde $f_{y p d}$ se corresponde $\operatorname{con} f_{y p} / \gamma_{s p}$ y $\gamma_{s p}=1,15$.

En este trabajo se consideran dos niveles de armado pasivo, $A_{s}$ y $A_{s}^{\prime}$, colocados a una distancia $d$ y $d$ ' de la fibra superior respectivamente, y un nivel para la armadura activa, $A_{p}$, localizado a una distancia $d_{p}$ desde la fibra superior -Figura $3-$. Tal y como se ha mencionado anteriormente, la deformación en estado último de rotura para cada fibra de la sección puede ser expresada en términos de la posición de la fibra neutra $x$ de acuerdo con [13]. De esta manera, haciendo uso de la composición de funciones matemáticas, [13], [17] y [18] pueden ser combinadas tal y como hace Gil-Martin et al. (10) teniendo en cuenta las deformaciones de cada uno de los niveles de armado:

$$
\begin{aligned}
& \varepsilon_{s}^{\prime}(x)=\varepsilon(d, x) \\
& \varepsilon_{s}(x)=\varepsilon(d, x) \\
& \varepsilon_{p}(x)=\varepsilon\left(d_{p}, x\right)-\varepsilon_{p(0)} \\
& \sigma_{s}^{\prime}(x)=\sigma_{s}^{\prime}\left(\varepsilon_{s}^{\prime}(x)\right)=\left(\sigma_{s}^{\prime} \circ \varepsilon_{s}^{\prime}\right)(x) \\
& \sigma_{s}(x)=\sigma_{s}\left(\varepsilon_{s}(x)\right)=\left(\sigma_{s} \circ \varepsilon_{s}\right)(x) \\
& \sigma_{p}(x)=\sigma_{p}\left(\varepsilon_{p}(x)\right)=\left(\sigma_{p} \circ \varepsilon_{p}\right)(x)
\end{aligned}
$$

donde • significa composición de dos funciones matemáticas y $\varepsilon_{p(0)}$ es la deformación en la armadura activa debida al tesado inicial, $\varepsilon_{p o}$, teniendo en cuenta las pérdidas de tesado a tiempo infinito más la deformación de descompresión del hormigón a nivel de la fibra de armadura activa, $\varepsilon_{c p}$ :

$$
\varepsilon_{p(0)}=\varepsilon_{c p}+\beta \varepsilon_{p o}
$$

\subsection{Ecuaciones de equilibrio}

Una vez que son conocidos los modelos de tensión - deformación de los materiales y teniendo en cuenta que la distribución de deformaciones unitarias a lo largo de la sección viene definida por de la posición de la fibra neutra, $x$, las ecuaciones de equilibrio pueden ser planteadas como se indica en [21]. Las tensiones normales a lo largo de la sección deben equilibrar la fuerza axil $N$ y al momento flector $M$. Tomando momentos respecto al centro de gravedad de la sección, las ecuaciones de equilibrio quedan:

$$
\begin{aligned}
& N=N_{c}(x)+A_{s} \sigma_{s}(x)+A_{s}^{\prime} \sigma_{s}^{\prime}(x)+A_{p} \sigma_{p}(x) \\
& M=N_{c}(x)\left(h_{c q}-Z_{c}(x)\right)+A_{s} \sigma_{s}(x)\left(h_{c g}-d\right)+A_{s}^{\prime} \sigma_{s}^{\prime}(x)\left(h_{c g}-d^{\prime}\right)+A_{p} \sigma_{p}(x)\left(h_{c q}-d_{p}\right)
\end{aligned}
$$

donde $h_{c g}$ es la posición del centro de gravedad de la sección -Figura 2- con respecto a la fibra superior y $z_{c}$ es la posición del centro de compresiones del hormigón también con respecto a la fibra superior, definida como:

$$
Z_{c}(x)=\left\{\begin{array}{lll}
\int_{0}^{\lambda x} \xi b(\xi) d \xi & \\
\frac{\int_{0}^{\lambda x} b(\xi) d \xi}{\lambda} & \text { si } & 0 \leq x<h / \lambda \\
h_{c g} & \text { si } & x \geq h / \lambda
\end{array}\right.
$$

Tiene sentido comentar en este punto que, debidos a los fenómenos de fluencia y retracción del hormigón y relajación del acero, los planos de tensiones y deformaciones nulas de la sección no coinciden a tiempo infinito. Estos hechos no han sido tenidos en cuenta al plantear las tensiones en el hormigón y en las armaduras como función de la posición de la fibra neutra, $x$. Dado que el procedimiento planteado en este trabajo es una técnica de predimensionamiento, estos fenómenos deberán ser tenidos en cuenta una vez finalizado el diseño de la sección al completo.

\section{DIAGRAMAS RSD: INTRODUCCIÓN DE LAS VARIABLES $A_{P}$ Y $D_{P}$}

Presentadas las ecuaciones de equilibrio en Eq. [21], la resolución del problema de diseño del armado pasivo de una sección en estado límite último -supuestos conocidos $A_{p} \mathrm{y}$ $d_{p}$ - conlleva la determinación de la posición de la fibra neutra $x$ y las áreas de armado pasivo superior $A_{\mathrm{s}}$ ' e inferior $A_{\mathrm{s}}$. Imponiendo equilibrio de momentos a nivel de las armaduras superior e inferior, Hernández-Montes et al. (1) expresan $A_{s}^{\prime}$ y $A_{s}$ como función de $x$, de tal manera que se pueden representar gráficamente todas las soluciones de armado en función de la posición de la fibra neutra en los denominados diagramas RSD. Estos diagramas permiten la selección de la solución óptima -mínima- de armado pasivo.

Ahora bien, si el problema de diseño implica también la determinación de los parámetros $A_{p} \mathrm{y} d_{p}$, dichos parámetros pueden ser introducidos en las ecuaciones de la metodología RSD, obteniendo:

$$
A_{s}\left(x, A_{p}, d_{p}\right)=\frac{-M_{d}+N_{d}\left(h_{g c}-d^{\prime}\right)-N_{c}(x)\left(Z_{c}(x)-d^{\prime}\right)-A_{p} \cdot \sigma_{p}(x)\left(d_{p}-d^{\prime}\right)}{\sigma_{s}(x)\left(d-d^{\prime}\right)}
$$




$$
A_{s}^{\prime}\left(x, A_{p}, d_{p}\right)=\frac{M_{d}+N_{d}\left(d-h_{g c}\right)-N_{c}(x)\left(d-Z_{c}(x)\right)-A_{p} \cdot \sigma_{p}(x)\left(d-d_{p}\right)}{\sigma_{s}^{\prime}(x)\left(d-d^{\prime}\right)}
$$

A través de [1] el valor de $A_{p}$ se puede expresar en función de la fuerza de tesado inicial $P_{0}$. En este trabajo, los pares de valores $A_{p}-d_{p}$ a introducir en [23] y [24] se determinarán a partir del diagrama de Magnel y haciendo uso de la representación RSD se obtendrá el mínimo de armado pasivo para cada uno de esos pares.

\section{METODOLOGÍA DEL PROCEDIMIENTO DE OPTIMIZACIÓN}

Una vez explicados los diagramas de Magnel para secciones de hormigón posteso, presentadas las consideraciones relativas al dimensionamiento en rotura de secciones en flexión compuesta así como los diagramas RSD con la inclusión de las variables $A_{p}$ y $d_{p}$, en este apartado se expone el procedimiento de optimización ideado. La Figura 4 presenta esquemáticamente dicho procedimiento.

Conocidas la geometría del elemento, las acciones a las que se ve sometido el mismo y las características del hormigón de la sección, el diagrama de Magnel puede construirse mediante la representación gráfica de las inecuaciones presentadas en [7] - [11].

Dado que los cordones de armadura activa se comercializan con áreas nominales concretas, la región válida del diagrama de Magnel se reducirá a pares de valores $A_{p}-d_{p}$ discretos, es decir, puntos. Así pues, definido un conjunto de valores $A_{p}-d_{p}$ relativos a un área nominal de armadura activa, $A_{p n o m}$, y a un paso determinado para el valor de la posición de la armadura activa, $d_{p s t e p}$, se seleccionan aquellos puntos que se encuentran dentro de la región válida de Magnel.

Cada par $A_{p}-d_{p}$ dentro de la región válida del diagrama de Magnel es ahora introducido en el procedimiento RSD -[23] y [24]- obteniendo así unos valores óptimos de $A_{s}$ y $A_{s}^{\prime}, \sin$ tener en cuenta consideraciones de cuantía mínima. Finalmente, cuando todos los pares $A_{p}-d_{p}$ han sido analizados, de entre todas las soluciones posibles se seleccionará aquella que minimice el coste total de armado, por metro lineal. Para determinar dicho coste, se han considerado los precios unitarios expuestos en la Tabla 2 según la «Base de precios tipo para los proyectos de plataforma (BPGP)» de ADIF (11). Los precios expuestos incluyen toda la mano de obra y medios auxiliares necesarios para la ejecución de las armaduras en obra.

Tabla 2. Costes unitario considerados para los aceros pasivo y activo.

\begin{tabular}{|c|c|}
\hline Tipo Acero & Coste (€/mm $\mathbf{~})$ \\
\hline Pasivo & 0,00683 \\
\hline Activo & 0,02002 \\
\hline
\end{tabular}

Tal y como se ha mencionado anteriormente, la metodología propuesta en este trabajo es una herramienta de predimensionamiento que no pretende eliminar ningún tipo de iteración para ajustar el diseño, ya sea calculando las pérdidas efectivas de tesado -teniendo en cuenta la presencia de

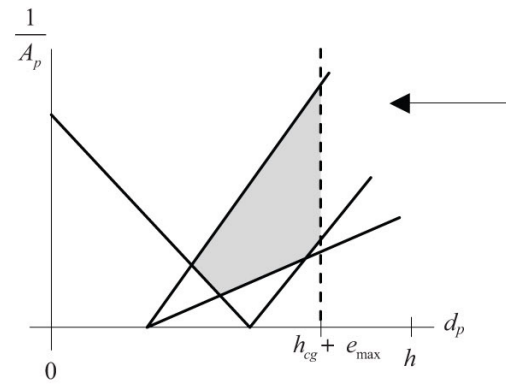

DIAGRAMA DE MAGNEL

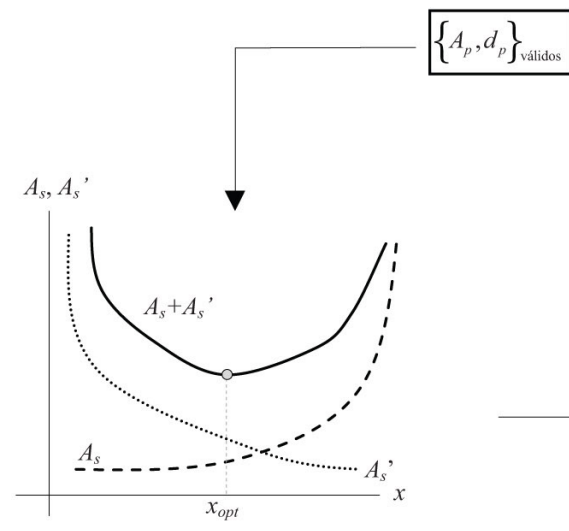

DIAGRAMA RSD PARA CADA $A_{p}-d_{p}$ VÁLIDO

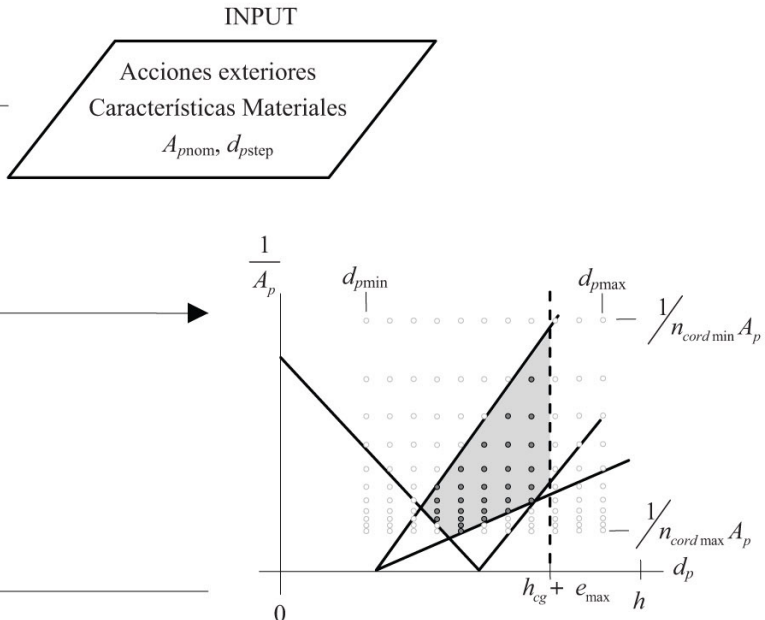

SELECCIÓN DE PARES $A_{p}-d_{p}$ VÁLIDOS

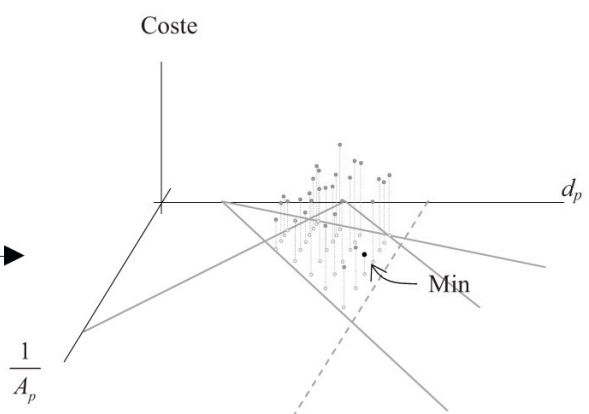

OBTENCIÓN DEL MÍNIMO ABSOLUTO

Figura 4. Esquema del procedimiento de optimización. 
armadura pasiva- o evaluando las condiciones de tensiones expuestas anteriormente con las propiedades geométricas de la sección neta y homogeneizada.

\section{EJEMPLO}

Sea una sección monolítica, en centro de vano, de una pieza de hormigón pretensado adherente con armadura postesa con unas dimensiones y solicitaciones como se muestran en la Figura 5. La clase de exposición ambiental será XC2 según EC2 (7). Las características del acero pasivo y activo se resumen en las Tablas 3 y 4.

Tabla 3. Características del acero pasivo.

\begin{tabular}{|c|c|}
\hline Acero pasivo & B 50o S \\
\hline$f_{y}$ & $500 \mathrm{MPa}$ \\
\hline$E_{s}$ & $200.000 \mathrm{MPa}$ \\
\hline
\end{tabular}

Tabla 4. Características del acero activo.

\begin{tabular}{|c|c|}
\hline Acero activo & Y1860S7 $\mathbf{\Phi ~ 1 5 . 2 ~}$ \\
\hline Área cordón, $A_{p \text { nom }}$ & $140 \mathrm{~mm}^{2}$ \\
\hline$f_{p k}$ & $1.860 \mathrm{MPa}$ \\
\hline$E_{p}$ & $190.000 \mathrm{MPa}$ \\
\hline
\end{tabular}

Para la estimación de las pérdidas instantáneas y diferidas de la fuerza de pretensado se estima que $\alpha=0,90$ y $\beta=0,75$. Por otra parte, se toma que el momento hiperestático del pretensado es un 30\% del isóstático y de signo contrario para la sección considerada. Para la obtención del armado óptimo el paso para la variable $d_{p}$ en el diagrama de Magnel $-d_{p s t e p}$-ha sido considerado igual a $10 \mathrm{~mm}$.

La Figura 6 muestra el diagrama de Magnel del ejemplo, con la delimitación de la región de pares $A_{p}-d_{p}$ válidos. La condición expresada en [11] nos da un valor de 4805,1 mm, muy por encima del valor de $d_{p}$ máximo tomado de $930 \mathrm{~mm}$.

En la Figura 7(a) se presentan el análisis de coste de las soluciones de armado obtenidas mediante la introducción en el RSD de todos los pares $A_{p}-d$ válidos de la Figura 6 y la Figura 7(b) muestra el diagrama RSD correspondiente a la solución óptima. Los valores de la posición y área de pretensado, posición de la fibra neutra y de las áreas de armadura pasiva inferior y superior se muestran para el valor óptimo en la Tabla 5 .

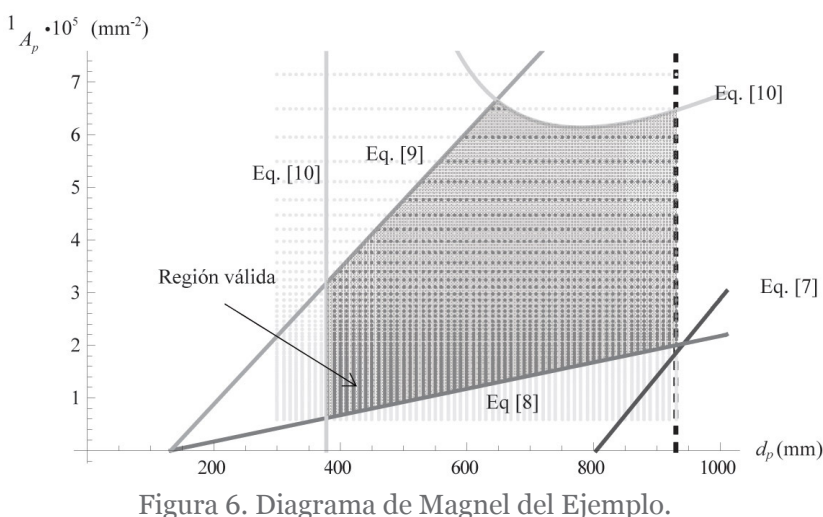

Se observa cómo, por la tipología de sección tomada, la cantidad de armadura superior necesaria para verificar el equilibrio en agotamiento es mínima, teniendo que disponer en este caso una cuantía mínima de armadura superior del orden de $5100 \mathrm{~mm}^{2}$-aplicando las prescripciones de la Tabla 42.3.5 de EHE-o8 (6)-. Además, el rango de posiciones de la fibra neutra $x$ donde existen soluciones de armado pasivo se sitúa en la cabeza superior de la sección, que al ser ésta de un espesor importante, corrobora la innecesaria presencia de armado pasivo superior. En este ejemplo, la excentricidad del pretensado correspondiente a la solución óptima es la máxima permisible ya que se ha tomando un recubrimiento de 70 $\mathrm{mm}$ desde la fibra inferior de la sección.

\section{CONCLUSIONES}

Los métodos tradicionales de diseño de elementos de hormigón armado y pretensado se han visto superados por las nuevas técnicas de cálculo numérico, la capacidad computacional y los nuevos planteamientos de optimización a la hora de decidir las cuantías de armado. Aunando estas nuevas posibilidades con el diseño de hormigón posteso tradicional, se ha aplicado la metodología de los diagramas RSD al método de diseño por medio de los diagramas de Magnel para la sección más desfavorable de un elemento de hormigón posteso, pudiendo así obtener el valor mínimo de acero necesario en la etapa de diseño.

Con la metodología desarrollada se presenta un procedimiento de predimensionamiento que agrupa tanto el estado límite último como el estado límite de servicio para la sección más
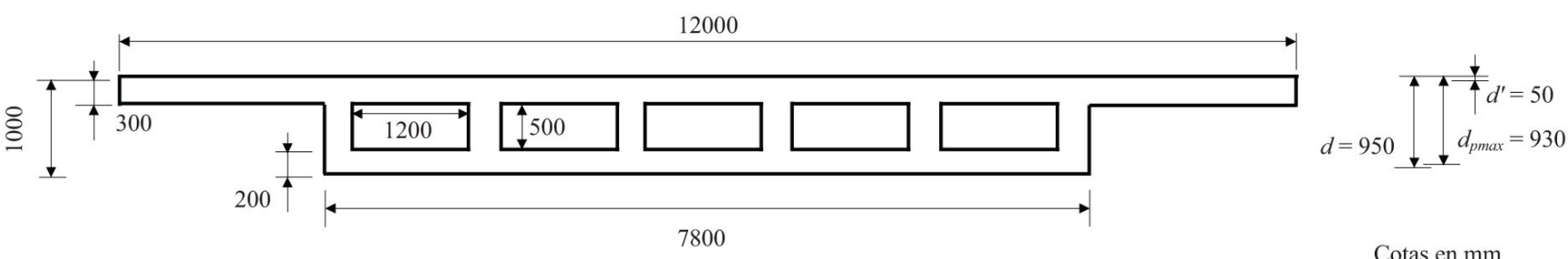

7800

Características del Hormigón:

$f_{c k}=45 \mathrm{MPa}$

$f_{c i}=0,95 f_{c k} \mathrm{MPa}$
Cargas exteriores:

$$
\begin{aligned}
& M_{p p}=6130 \mathrm{KN} \cdot \mathrm{m} \\
& M_{\text {cuasip }}=7810 \mathrm{KN} \cdot \mathrm{m} \\
& M_{\text {frec }}=11860 \mathrm{KN} \cdot \mathrm{m} \\
& N_{u}=0 \mathrm{KN} \\
& M_{u}=21910 \mathrm{KN} \cdot \mathrm{m}
\end{aligned}
$$

Figura 5. Sección e input para el ejemplo. 
Tabla 5. Solución de armado óptimo para el Ejemplo.

\begin{tabular}{|c|c|c|c|c|c|c|}
\hline$d_{p}(\mathrm{~mm})$ & $A_{p}\left(\mathrm{~mm}^{2}\right)$ & $\mathrm{N}^{0}$ cables & $x(\mathrm{~mm})$ & $A_{s}\left(\mathrm{~mm}^{2}\right)$ & $A_{s}^{\prime}\left(\mathrm{mm}^{2}\right)$ & Coste $(€ / \mathrm{m})$ \\
\hline 930 & 15540 & 111 & 124 & 17583 & 339,1 & 433.516 \\
\hline
\end{tabular}

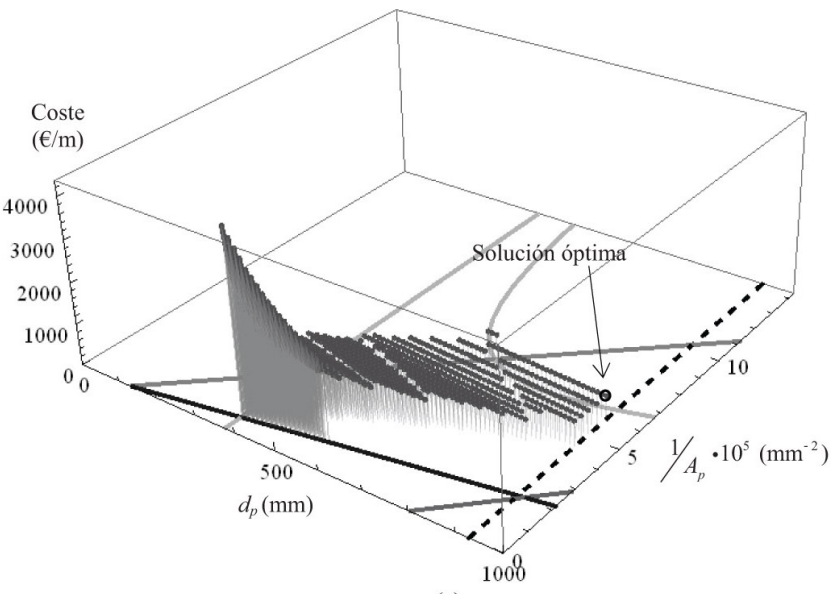

(a)

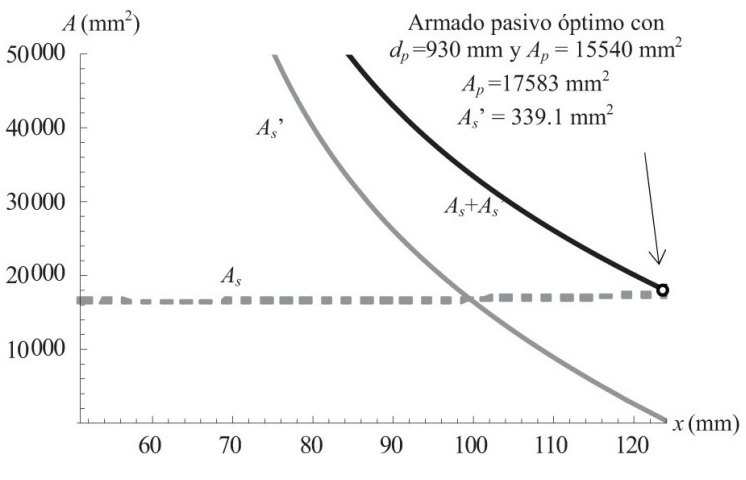

(b)

Figura 7. (a) Representación grática del coste de armado tras el RSD para los pares válidos $A_{p}-d_{p}$; (b) diagrama RSD correspondiente a la solución óptima.

desfavorable, pudiendo así optimizar el coste de área total de acero pasivo -entendido como la suma de $A_{s}+A_{s}$ '- y el coste del área de acero activo $A_{p} \mathrm{y}$, por consiguiente, conseguir un ahorro económico al obtener el valor óptimo de entre todas las soluciones posibles.

Se ha planteado un ejemplo mostrando los valores óptimos de posición de la armadura activa, $d_{p}$, áreas de acero pasivo
$A_{\mathrm{s}} \mathrm{y} A_{\mathrm{s}}^{\prime} \mathrm{y}$ de área de acero activo $A_{p}$. Observando los resultados obtenidos es evidente que esta metodología es una herramienta útil y versátil con la que el ingeniero puede afrontar el problema de diseño de elementos postesos.

Por la tipología de la sección tomada en el ejemplo, es decir, una sección con gran cabeza de compresión, se puede observar que el resultado de este método propuesto coincide con los resultados esperables con el método tradicional, sólo teniendo que disponer la cuantía mínima de armadura superior en la sección más solicitada. Para secciones en las que no se cuente con una gran cabeza de compresión, como en secciones tipo $\mathrm{T}$ invertida, esta metodología de optimización haría que los resultados difirieran de los obtenidos con la metodología tradicional. Así pues, el ingeniero dispondría de un criterio decisión basado en la optimización económica de armados.

Aunque el procedimiento se ha presentado aquí para una sección concreta, éste es extrapolable a otras secciones transversales de la viga lo que permitiría optimizar la posición de la armadura activa y el área total de acero a lo largo de toda la viga.

Puesto que los diagramas de Magnel no tienen en cuenta la presencia de la armadura pasiva y las inecuaciones presentes en el mismo se han planteado con las propiedades geométricas de la sección bruta, el procedimiento descrito en este trabajo requerirá de, al menos, una iteración para comprobar que el predimensionamiento es correcto y que las pérdidas de tesado fueron bien estimadas. Partiendo del predimensionamiento obtenido con este procedimiento, el diseño podrá afinarse en mayor medida posteriormente.

\section{AGRADECIMIENTOS}

Los autores agradecen sinceramente la contribución realizada por los revisores con las observaciones realizadas. El presente trabajo ha sido parcialmente financiado por el Ministerio de Economía y Competitividad del Gobierno de España bajo el proyecto de investigación IPT-2011-1485-4200oo. El segundo autor es becario predoctoral del Ministerio de Educación (Beca FPU AP 2010-3707).

\section{REFERENCIAS}

(1) Hurst, M.K. (1998). Prestressed Concrete Design ( $2^{\text {nd }}$ Ed.). New York: E\&FN Spon.

(2) Hernández-Montes, E., Gil-Martín, L.M., Aschheim, M. (2005). Design of concrete members subjected to uniaxial bending and compression using reinforcement sizing diagrams. ACI Structural Journal, 102: 150-158.

(3) Hernández-Montes, E., Gil-Martín, L.M., Pasadas-Fernández, M., Aschheim M. (2008). Theorem of optimal reinforcement for reinforced concrete sections. Structural and Multidisciplinary Optimization, 36(5): 509-521.

(4) López-Martín, D, Carbonell-Márquez, J.F., Gil-Martín, L.M., Hernández-Montes, E. (2014). Eccentricity-based optimization procedure for strength design of RC sections under compression and in plane bending moment. Journal of Structural Engineering ASCE, 140(1), doi: http://dx.doi.org/10.1061/(ASCE)ST.1943-541X.0000794.

(5) Albajar-Molera, L. (2013). ELS en Estructuras Pretensadas. En Gil-Martín, L.M. (coord.) Problemas resueltos de elementos estructurales de Hormigón Armado y Pretensado según EHE-o8 y EC2, ${ }^{\text {a }}$ Ed. Cap. 12 (pp. 211-236). Madrid: Ibergarceta Publicaciones.

(6) Comisión Permanente del Hormigón. (2008). Instrucción de Hormigón Estructural. EHE o8. Madrid: Ministerio de Fomento. 
(7) European committee for Standaritztion. (2004). EN-1992-1-1. Eurocode 2: Design of Concrete Structures- Part 1-1. General Rules and Rules for Buildings. Bruselas: European committee for Standarization.

(8) Marí-Bernat, A.R., Aguado-de Cea, A., Agulló-Fité, L., Martínez-Abella, F., Cobo del Arco, D. (1999). Hormigón armado y pretensado. Ejercicios. Adaptado a la Instrucción EHE (pp. 305-322). Barcelona: Edicions UPC.

(9) Salguero, F., Romero, S., Melgar, S.G., Prat, F., Moreno, F. (2013). Las curvas tensión-deformación de hormigones ensayados bajo compresión uniaxial monotónica: una revisión sistemática. Informes de la Construcción, 65(529): 41-54, doi: http://dx.doi.org/10.3989/ic.11.114.

(10) Gil-Martín, L.M., López-Martín, D., Hernández-Montes, E., Aschheim, M. (2012). Dimensionamiento en rotura a flexión de secciones de hormigón armado. Un planteamiento compacto. Informes de la Construcción, 64(528): 497-505, http:// dx.doi.org/10.3989/ic.11.050.

(11) ADIF. (2011). Sistema de Gestión. Tomo V: Base de precios tipo general para los proyectos de plataforma. Edición junio 2011, versión 2. Madrid: Administrador de Infraestructuras Ferroviarias. 